\title{
Recognition and Optimization of Loop-Carried Stream Reusing of Scientific Computing Applications on the Stream Processor
}

\author{
Ying Zhang, Gen Li, and Xuejun Yang \\ Institute of Computer, National University of Defense Technology, 410073 Changsha China \\ zhangying@nudt.edu.cn
}

\begin{abstract}
Compared with other stream applications, scientific stream programs are usually constrained by memory access. Loop-carried stream reusing means reusing streams across different iterations and it can improve the locality of SRF greatly. In the paper, we present algorisms to recognize loop-carried stream reusing and give the steps to utilize the optimization after analyzing characteristics of scientific computing applications. Then we perform several representative microbenchmarks and scientific stream programs with and without our optimization on Isim. Simulation results show that stream programs optimized by loop-carried stream reusing can improve the performance of memory-bound scientific stream programs greatly.
\end{abstract}

\section{Introduction}

Now conventional architecture has been not able to meet the demands of scientific computing[1][2]. In all state-of-the-art architectures, the stream processor[3](as shown in fig. 1) draws scientific researchers' attentions for its processing computation-intensive applications effectively[4-8]. Compared with other stream applications, scientific computing applications have much more data, more complex data access methods and more strong data dependence.

The stream processor has three level memory hierarchies[12] - local register files (LRF) near ALUs exploiting locality in kernels, global stream register files (SRF) exploiting producer-consumer locality between kernels, and streaming memory system exploiting global locality. The bandwidth ratios between three level memory hierarchies are large. In Imagine[9][10], the ratio is 1:13:218. As a result, how to enhance the locality of SRF and LRF and consequently how to reduce the chip-off memory traffics become key issues to improve the performance of scientific stream programs constrained by memory access. Fig. 2 shows a stream flows across three level memory hierarchies during the execution of a stream program. First, the stream is loaded from chip-off memory into SRF and distributed into corresponding buffer. Then it is loaded from SRF to LRF to supply operands to a kernel. During the execution of the kernel, all records participating in kernel and temporary results are saved in LRF. After the kernel is finished, the records are stored back to SRF. If there is producer-consumer locality between this kernel and its later kernel, the stream is saved in SRF. Otherwise, it is stored back to chip-off memory. 
Loop-carried stream reusing means reusing streams across different iterations and it can improve the locality of SRF. In the paper, we present algorisms to recognize loop-carried stream reusing and give steps to use our method to optimize stream programs according to the analysis of typical scientific computing applications. We give the recognition algorism to decide what applications can be optimized and the steps how to utilize loop-carried stream reusing to optimize stream organization. Then we perform several representative microbenchmarks and scientific stream programs with and without our optimization on a cycle-accurate stream processor simulator. Simulation results show that the optimization method can improve scientific stream program performance efficiently.

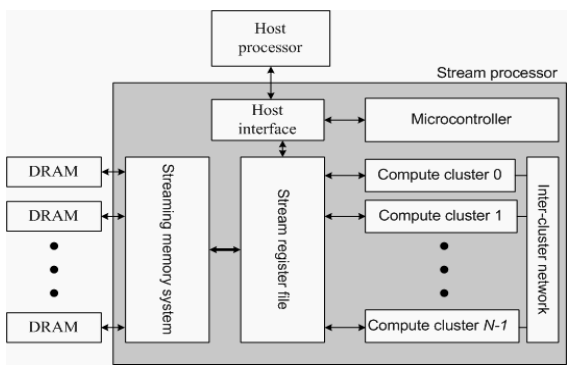

Fig. 1. Block diagram of a stream processor

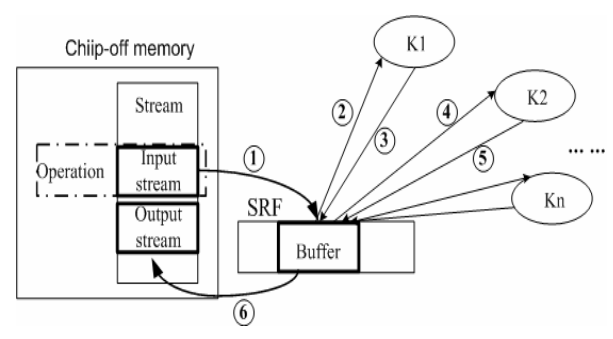

Fig. 2. Stream flowing across the memory system

\section{Loop-Carried Stream Reusing}

Loop-carried stream reusing is defined as that between neighboring loop iterations of a stream-level program, input or output streams of kernels in the first iteration can be used as input streams of kernels in the second iteration. If input streams are reused, we call it loop-carried input stream reusing. Otherwise, we call it loop-carried output stream reusing. The essential of stream reusing optimization is to enhance the locality of SRF. Correspondingly, input stream reusing can enhance producer-producer locality of SRF while output stream reusing can enhance producer-consumer locality of SRF.

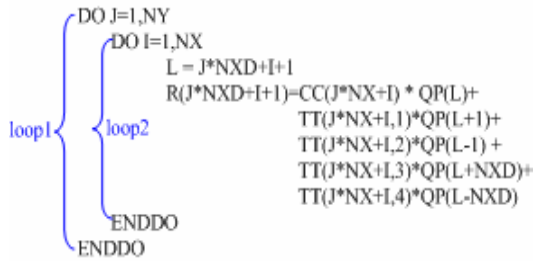

Fig. 3. Example code

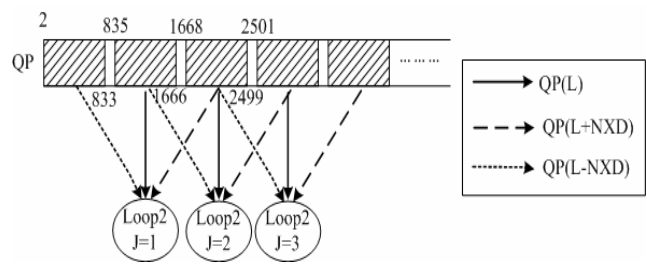

Fig. 4. Data trace of array QP references

Then we take code in fig. 3 from stream program MVM as example to depict our methods, where NXD equals to NX+2. In the paper, we let NX and NY equal to 832. 
Fig. 4 shows the data trace of $\mathrm{QP}(\mathrm{L}), \mathrm{QP}(\mathrm{L}+\mathrm{NXD})$ and $\mathrm{QP}(\mathrm{L}-\mathrm{NXD})$ participating in loop2 of fig. 3. QP(1668,2499) is $\mathrm{QP}(\mathrm{L}+\mathrm{NXD})$ of loop2 when $\mathrm{J}=1$, QP(L) of loop2 when $\mathrm{J}=2$, and QP(L-NXD) of loop2 when $\mathrm{J}=3$. So, stream QP can be reused between different iterations of loop1. If QP $(1668,2499)$ is organized as a stream, it will be in $\mathrm{SRF}$ after loop2 with $\mathrm{J}=1$ finishes. Consequently, when loop2 with $\mathrm{J}=2$ or $\mathrm{J}=3$ running, it doesn't get stream QP $(1668,2499)$ from chip-off memory but SRF.

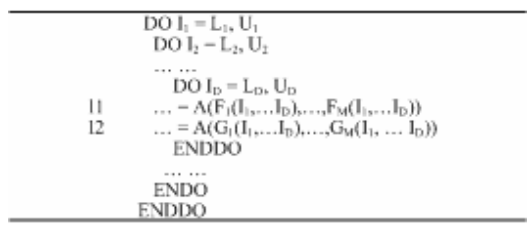

Fig. 5. A D-level nested loop

\subsection{Recognizing Loop-Carried Stream Reusing}

Fig. 5 shows a generalized perfect nest of D loops. The body of the loop nest reads elements of the m-dimensional array A twice. In the paper, we only consider linear subscription expressions, and the ith dimension subscription expression of the first array $\mathrm{A}$ reference is denoted as $F_{i}=\sum C_{i, j} * I_{j}+C_{i, 0}$, where $\mathrm{I}_{\mathrm{j}}$ is an index variable, $1 \leq j \leq D, \mathrm{C}_{\mathrm{i}, \mathrm{j}}$ is the coefficient of $\mathrm{I}_{\mathrm{j}}, 1 \leq j \leq D$, and $\mathrm{C}_{\mathrm{i}, 0}$ is the remaining part of the subscript expression that does not contain any index variables. Correspondingly, the ith dimension subscription expression of the second array A reference is denoted as $G_{i}=\sum C_{i, j}^{\prime}{ }^{*} I_{j}+C_{i, 0}^{\prime}$. If in the Pth level loop, the data trace covered by the leftmost $\mathrm{Q}$ dimensions ${ }^{1}$ of one array $A$ read references in the nest with $\mathrm{I}_{\mathrm{P}}=\mathrm{i}$ is the same to that of the other array A read references in the nest with $I_{P}=i+d$, where $d$ is a const, and they are different to each other in the same nest, such as the data trace of array QP in loop2 in fig. 3, the two array A read references can be optimized by input stream reusing in respect of loop P and dimension Q. Then we give the algorism of Recognizing Loopcarried Input Stream Reusing.

RLISR. Two array references in the same loop body can be optimized by input stream reusing in respect of loop $\mathrm{P}$ and dimension $\mathrm{Q}$ if:

(1) when $M=1$, i.e. array A is a 1-D array, the subscript expressions of two array A references can be written as $F_{1}=\sum C_{1, j} * I_{j}+C_{1,0}$ and $G_{1}=\sum C_{1, j}^{\prime} *_{j}+C_{1,0}^{\prime}$ respectively, and $\mathrm{Q}=1$ now. The coefficients of $\mathrm{F} 1$ and $\mathrm{G} 1$ should satisfy following formulas:

$$
\begin{gathered}
\forall j:\left((1 \leq j \leq D) \wedge C_{1, j}=C_{1, j}^{\prime}\right) \\
C_{1,0}=C_{1,0}^{\prime} \pm d * C_{1, P}^{\prime} \\
\forall j:\left((1 \leq j \leq D) \wedge\left(C_{1, j} \geq \sum_{j=1}^{D} C_{1, j} *\left(U_{j}-L_{j}\right)\right)\right.
\end{gathered}
$$

\footnotetext{
${ }^{1}$ In this paper, we assume the sequence of memory access is the leftmost dimension first just like as that in FORTRAN.
} 
Formula (1a) and (1b) ensure when the loop indices of outmost P-1 loops are given and the loop body passes the space of innermost D-P loops, the data trace of one array A read reference in the nest with $I_{P}=i$ is the same to that of the other array $A$ read references in the nest with $\mathrm{I}_{\mathrm{P}}=\mathrm{i}+\mathrm{d}$. $\mathrm{d}$ is a const and we specify $\mathrm{d}=1$ or 2 according to[13]. When $I_{1}, \ldots, I_{P-1}$, are given and $I_{P}, \ldots, I_{D}$ vary, formula(1c) restricts the data trace of one array A references in the nest with $\mathrm{I}_{\mathrm{P}}=\mathrm{i}$ from overlapping that of the other in the nest with $I_{P}=i+d$. This formula ensures the characteristic of stream process, i.e. data as streams is loaded into the stream processor to process and reloaded into SRF in batches after process. For the stream processor, the cost of random access records of streams is very high.

(2) when $M \neq 1$, i.e. array A is a multi-dimensional array, the subscript expressions of two array A read references should satisfy following conditions:

(d) the Qth dimension subscript expression of one array is gotten by translating the index $I_{P}$ in the dimension subscript expression of the other array by $d$, i.e. $G_{Q}=F_{Q}\left(I_{P} \pm d\right)$, and,

(e) all subscript expressions of one array A reference are the same with those of the other except the Qth dimension subscript expression, i.e. $\forall i\left((i \neq Q) \wedge\left(F_{i}=G_{i}\right)\right)$, and,

(f) for the two array A reference, the innermost index variable in one subscript expression will not appear in any righter dimension subscript expressions, i.e.

$$
\forall i\left(\ni j\left(C_{i, j} \neq 0 \wedge \forall j^{\prime}\left(j^{\prime}>j \wedge C_{i, j^{\prime}}=0\right)\right) \rightarrow \forall i^{\prime}\left(i^{\prime}>i \wedge C_{i^{\prime}, j}=0\right)\right) \wedge \forall i\left(\ni j\left(C_{i, j} \neq 0 \wedge \forall j^{\prime}\left(j^{\prime}>j \wedge C_{i, j^{\prime}}=0\right)\right) \rightarrow \forall i^{\prime}\left(i^{\prime}>i \wedge C_{i^{\prime}, j}=0\right)\right) .
$$

It can be proved that data access trace of two array references decided by condition (2) satisfies condition (1), and when $\mathrm{U}_{\mathrm{j}} \mathrm{I}_{\mathrm{j}}$ is large enough, they are equivalent.

The algorism of Recognizing Loop-carried Output Stream Reusing is similar to RLISR except that reusing stream mustn't change original data dependence. Then we give the RLOSR algorism without detailed specifications.

RLOSR. We denote the subscript expressions of read references as $F_{i}$ and those of write references as $\mathrm{G}_{\mathrm{i}}$. Two array references in loop body can be optimized by output stream reusing in respect of loop $\mathrm{P}$ and dimension $\mathrm{Q}$ if:

(3) when $M=1$, the coefficients of F1 and G1 should satisfy following formulas:

$$
\begin{gathered}
\forall j:\left((1 \leq j \leq D) \wedge C_{1, j}=C_{1, j}^{\prime}\right) \\
C_{1,0}=C_{1,0}^{\prime}+d^{*} C_{1, P}^{\prime} \\
\forall j:\left((1 \leq j \leq D) \wedge\left(C_{1, j} \geq \sum_{i=1}^{D} C_{1, j} *\left(U_{j}-L_{j}\right)\right)\right.
\end{gathered}
$$

(4) when $M \neq 1$, the subscript expressions of two array A read references should satisfy following formulas:

$$
\begin{gathered}
G_{Q}=F_{Q}\left(I_{P}+d\right) \\
\forall i\left((i \neq Q) \wedge\left(F_{i}=G_{i}\right)\right) \\
\forall i\left(\ni j\left(C_{i, j} \neq 0 \wedge \forall j^{\prime}\left(j^{\prime}>j \wedge C_{i, j^{\prime}}=0\right)\right) \rightarrow \forall i^{\prime}\left(i^{\prime}>i \wedge C_{i, j}=0\right)\right) \wedge \forall i\left(\ni j\left(C_{i, j} \neq 0 \wedge \forall j^{\prime}\left(j^{\prime}>j \wedge C_{i, j^{\prime}}=0\right)\right) \rightarrow \forall i^{\prime}\left(i^{\prime}>i \wedge C_{i^{\prime}, j}=0\right)\right)
\end{gathered}
$$

\subsection{Optimizing Loop-Carried Stream Reusing}

Then we give the step of using input stream reusing method to optimize stream organization. 
Step A. Organize different array A references in the innermost D-P loops as stream A1 and A2 according their data traces.

Step B. Organize all operations on array A in the innermost D-P loops as a kernel.

Step C. Organize all operations in the outmost P loops as stream-level program.

When the nest with $\mathrm{I}_{\mathrm{P}}=\mathrm{i}$ of loop $\mathrm{P}$ in stream-level program operates on stream A1 and A2, one of them has been loaded into SRF by the former nest, which means that the kernel doesn't get it from chip-off memory but SRF. From the feature of the stream processor architecture, we can know the time to access chip-off memory is much larger than that to access SRF, so the method of stream reusing can improve stream program performance greatly.

The steps to use output stream reusing are analogous to steps above.

In stream program MVM unoptimized, we organize different array QP read in loop1 as three streams according their data trace ,and. organize operations in loop1 as a kernel The length of each stream is $832 * 832$. When running, the stream program must load these three streams into SRF, the total length of which is $692224 * 3$, nearly three times of that of array QP.

By the stream reusing method above, we organize different array QP read references in loop2 as three streams according their own data trace, organize operations in loop2 as a kernel, and organize operations in loop1 except loop2 as stream-level program. Thus there would be $832 * 3$ streams in the stream program loop1, and the length of each is 832. So in stream program loop1, stream QP(L), $\mathrm{QP}(\mathrm{L}+\mathrm{NXD})$ and $\mathrm{QP}(\mathrm{L}-\mathrm{NXD})$ of neighboring iterations can be reused. As a result, the stream program only need load 832 streams with the length of 832 from chip-off memory to SRF, the total length of which is 692224 , nearly $1 / 3$ of that of unoptimized program.

\section{Experiment}

We compare the performance of microbenchmarks and several scientific applications optimized and unoptimized by stream reusing. All applications are run on a cycleaccurate simulator for a single-node Imagine stream processor, Isim[9][10].

Table 1 summarizes the test programs used for evaluation. Microbenchmarks listed in the upper half of the table stress particular aspects of loop-carried stream reusing, e.g., if there is an input stream reusing between adjacent loop nests in respect of loop 2 and dimension 2, the benchmark is named P2Q2d1. All microbenchmarks are stream programs of applications in fig. 6 in FORTRAN code. P2Q2d1, P3Q3d1, $\mathrm{P} 3 \mathrm{Q} 3 \mathrm{~d} 1 \mathrm{O}$ and P3Q3d2 are corresponding stream programs of 6(a), 6(b), 6(c) and

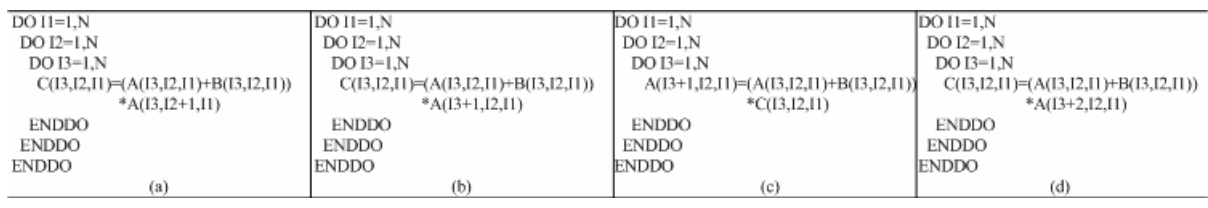

Fig. 6. FORTRAN code of applications to be optimized 
Table 1. Benchmark programs

\begin{tabular}{l|l} 
Name & Description \\
\hline P2Q2d1 & $\begin{array}{l}\mathrm{P}=\mathrm{Q}=2, \mathrm{~d}=1, \text { and optimized by input stream reusing. } \\
\text { same application as P2Q2d1 except that we don't optimize it by stream reusing but } \\
\text { organize array references of the innermost } 2 \text { loops as streams }\end{array}$ \\
P2Q2d13 & $\begin{array}{l}\text { same as P2Q2d112 except that array references of all 3 loops are organized as streams } \\
\mathrm{P}=\mathrm{Q}=3, \mathrm{~d}=1, \text { and optimized by input stream reusing }\end{array}$ \\
$\mathrm{P} 3 \mathrm{Q} 3 \mathrm{~d} 1$ & $\begin{array}{l}\text { same as P3Q3d1 except that it is optimized by output stream reusing } \\
\text { P3Q3d1O } \\
\text { s3Q3d2 } \\
\text { same as P3Q3d1 except that d=2 }\end{array}$ \\
QMR. & $\begin{array}{l}\text { ab. of QMRCGSTAB, a subspace method to solve large nonsymmetric sparse linear } \\
\text { systems[14] whose coefficient array size is } 800 * 800\end{array}$ \\
MVM & $\begin{array}{l}\text { a subroutine of a hydrodynamics application and computing band matrix } \\
\text { multiplication with the size of } 832 * 832 \\
\text { calculating the central difference of two-dimension array whose size is } 256 * 256\end{array}$
\end{tabular}
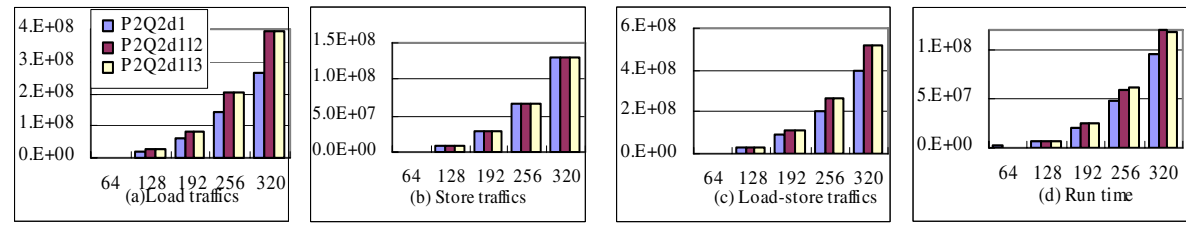

Fig. 7. With the increase of array size the performance of different stream implementations of the application in 6(a) in respect of memory traffics(bytes) and run time(cycles)
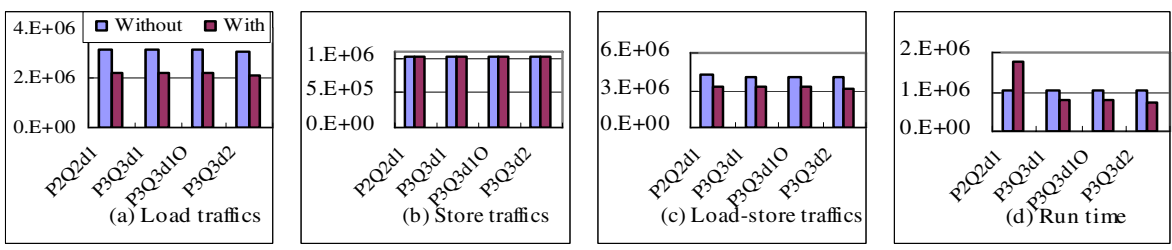

Fig. 8. Performance of $\mathrm{P} 2 \mathrm{Q} 2 \mathrm{~d} 1, \mathrm{P} 3 \mathrm{Q} 3 \mathrm{~d} 1, \mathrm{P} 3 \mathrm{Q} 3 \mathrm{~d} 1 \mathrm{O}$ and $\mathrm{P} 3 \mathrm{Q} 3 \mathrm{~d} 2$ with array size of 64 in respect of memory traffics(bytes) and run time(cycles)
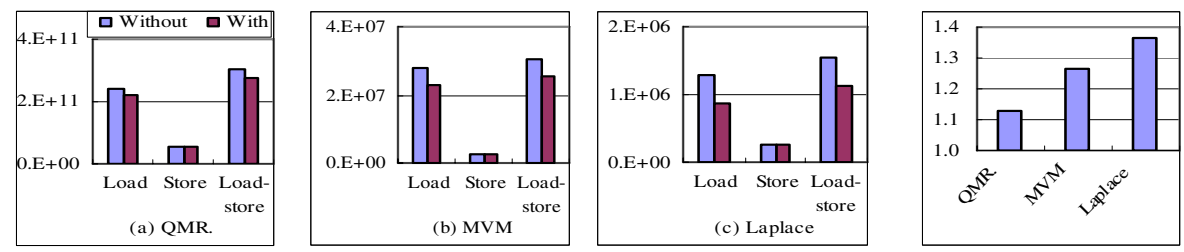

Fig. 9. Effects of stream reusing on the memory traffics of scientific programs

Fig. 10. Speedup of scientific programs with stream reusing

6(d), which is optimized by loop-carried stream reusing. P2Q2d112 and P2Q2d113 are corresponding stream programs of $6(\mathrm{a})$ without optimization. There are $2 * \mathrm{~N}$ out of $4 * \mathrm{~N}$ streams that can be reused as $\mathrm{N}$ stream in SRF in every microbenchmark except 
P2Q2d1, in which there are $2 * \mathrm{~N}^{2}$ out of $4 * \mathrm{~N}^{2}$ streams that can be reused as $\mathrm{N}^{2}$ stream in SRF . Scientific applications listed in the lower half of the table are all constrained by memory access. 14994 out of 87467 streams in QMR. can be reused as 4998 streams in SRF, 3 out of 8 streams in MVM can be reused as 1 stream in SRF, and 3 out of 5 streams in Laplace can be reused as 1 stream in SRF.

Fig. 7 shows the performance of different stream implementations of the application in 6(a) with the increase of array size. Fig. 7(a) shows chip-off memory load traffics, fig. 7(b) shows store traffics, fig. 7(c) shows the total chip-off memory traffics, and fig. 7(d) shows the run time of these implementations. In fig. 7(a), the load traffics of P2Q2d1 are nearly $2 / 3$ of the other two implementations whatever the array size is. This is because input loop-carried stream reusing optimization finds the loop-carried stream reusing, improves the locality of SRF and consequently reduces the load memory traffics. In fig. 7(b) the store traffics of different implementations are the same because there is only input stream reusing, which has no effect on store traffics. From fig. 7(c), we can see that because loop-carried stream reusing reuses 2 input streams as one stream in SRF, it cut down the total memory traffics obviously. In fig. 7(d), when the array size is 64 , the run time of P2Q2d1 is larger than the other two implementations. When the array size is 128 , the run time of P2Q2d1 is a little larger than the other two implementations. The reason for above is that when the array size is small, the stream length of $\mathrm{P} 2 \mathrm{Q} 2 \mathrm{~d} 1$ is much shorter than and the number of streams are larger than the other two implementations. As a result, the overheads to prepare to load streams from chip-off memory to SRF weigh so highly that they can't be hidden, including the time the host writes SDRs(Stream Descriptor Register) and MARs(Memory Access Register).With the increase of the array size, the run time of P2Q2d1 is smaller and smaller than the other two implementations. This is because with the increase of the stream length, the overheads to load streams into SRF weigh more and more highly and consequently the overheads to prepare loading streams can be hidden well. The memory traffics of P2Q2d1 are the least and consequently the $\mathrm{P} 2 \mathrm{Q} 2 \mathrm{~d} 1$ program performance is the highest.

Fig. 8 shows the performance of P2Q2d1, P3Q3d1, P3Q3d1O and P3Q3d2 with array size of 64. Fig. 8(a) shows chip-off memory load traffics, fig. 8(b) shows store traffics, fig. 8(c) shows the total chip-off memory traffics, and fig. 8(d) shows the run time of them. These applications are representative examples of loop-carried stream reusing. In fig. 8(a), 8(b) and 8(c), chip-off memory load, store and total traffics have similar characteristics as those in fig. 7. In fig. 8(d), the performance of all applications except $\mathrm{P} 2 \mathrm{Q} 2 \mathrm{~d} 1$ have been improved by stream reusing optimization. The reason for the reduction of $\mathrm{P} 2 \mathrm{Q} 2 \mathrm{~d} 1$ performance has been given above. The results show that these representative applications optimized by loop-carried stream reusing all get similar performance increase as that in fig. 7 .

Fig. 9 shows the effects of stream reusing on the memory traffics of scientific programs used in our experiments. Fig. 10 shows the speedup yielded by scientific applications with stream reusing over without. All these applications are optimized by input stream reusing. From results, we can see that because all these applications are constrained by memory access, the improvement of application performance brought by stream reusing is nearly in proportion to the amount of streams that can be reused. 


\section{Conclusion and Future Work}

In this paper, we give a recognition algorism to decide what applications can be optimized and the steps how to utilize loop-carried stream reusing to optimize stream organization. Several representative microbenchmarks and scientific stream programs with and without our optimization are performed on Isim which is a cycle-accurate stream processor simulator. Simulation results show that the optimization method can improve the performance of scientific stream program constrained by memory access efficiently.

In the future, we are devoted to developing more programming optimizations to take advantage of architectural features of the stream processor for scientific computing applications.

\section{References}

1. W. A. Wulf, S. A. McKee. Hitting the memory wall: implications of the obvious. Computer Architecture News, 1995. 23(1): 20-24.

2. D. Burger, J. Goodman, A. Kagi. Memory bandwidth limitations of future microprocessors. In Proceedings of the 23rd International Symposium on Computer Architecture, Philadelphia, PA, 1996.78-89.

3. Saman Amarasinghe, William. Stream Architectures. In PACT 2003, September 27, 2003.

4. Merrimac - Stanford Streaming Supercomputer Project, Stanford University, http://merrimac.stanford.edu/

5. William J. Dally, Patrick Hanrahan, et al., "Merrimac: Supercomputing with Streams", SC2003, November 2003, Phoenix, Arizona.

6. Mattan Erez, Jung Ho Ahn, et al., "Merrimac - Supercomputing with Streams", Proceedings of the 2004 SIGGRAPH GP^2 Workshop on General Purpose Computing on Graphics Processors, June 2004, Los Angeles, California.

7. Wang Guibin, Tang Yuhua, et al., "Application and Study of Scientific Computing on Stream Processor", Advances on Computer Architecture (ACA'06), August 2006, Chengdu, China.

8. Du Jing, Yang Xuejun, et al., "Implementation and Evaluation of Scientific Computing Programs on Imagine", Advances on Computer Architecture (ACA'06), August 2006, Chengdu, China.

9. MScott Rixner, Stream Processor Architecture. Kluwer Academic Publishers. Boston, MA, 2001.

10. Peter Mattson. A Programming System for the Imagine Media Processor. Dept. of Electrical Engineering. Ph.D. thesis, Stanford University, 2002

11. 11.Ola Johnsson, Magnus Stenemo, Zain ul-Abdin. Programming \& Implementation of Streaming Applications. Master's thesis, Computer and Electrical Engineering Halmstad University, 2005.

12. Ujval J. Kapasi, Scott Rixner, et al., Programmable Stream Processor, IEEE Computer, August 2003

13. Goff, G., Kennedy, K., and Tseng, C.-W. 1991. Practical dependence testing. In Proceedings of the SIGPLAN '91 Conference on Programming Language Design and Implementation. ACM, New York.

14. A Quasi-Minimal Residual Variant Of The Bi-Cgstab Algorithm For Nonsymmetric Systems (1994) T. F. Chan, E. Gallopoulos, V. Simoncini, T. Szeto, C. H. TongSIAM Journal on Scientific Computing. 